Vehicle System Dynamics, Vol.25 suppl., 1996, pp.139-151

\title{
Comparative Analyses of Three Types of Headway Control Systems for Heavy Commercial Vehicles
}

\author{
P.S. FANCHER, H. PENG, and Z. BAREKET
}

\begin{abstract}
SUMMARY
This paper compares design approaches for achieving a headway control functionality for trucks and buses. The approaches considered are fuzzy logic [1], $H_{\infty}$ [2], and a strategy based on headway range and its derivative (range-rate) [3]. For heavy vehicles, the control unit has a number of nonlinearities to compensate for, including full accelerator saturation, engine characteristics, and a limited deceleration capability as may be influenced by rolling resistance, aerodynamic drag, and retarder capabilities. Performance properties of the controllers are derived from simulations of basic operational situations such as closing-in on a preceding vehicle that is traveling at a slower speed or following a vehicle whose speed varies.
\end{abstract}

\section{HEADWAY-CONTROL CONSIDERATIONS}

The basic objective of headway control is to maintain a satisfactory separation between a preceding vehicle and a following vehicle equipped with a device that provides information concerning the range distance from the equipped vehicle to the preceding vehicle. Currently there exist infrared laser and microwave radar devices which provide range $(\mathrm{R})$ and range rate $(\mathrm{dR} / \mathrm{dt})$ information in a form that has been used for headway control purposes. See [4] and [5] for examples.

The desired headway range separation may be chosen to be a function of the speed of the preceding vehicle. This approach can aid in providing for the stability of a string (spatial sequence) of equipped vehicles. Also, it corresponds to traditional driving rules such as "allow one car length for each $10 \mathrm{mph}$." In this paper each controller uses a desired headway time $\mathrm{T}_{\mathrm{h}}=2 \mathrm{sec}$. (Two seconds has been demonstrated successfully in a heavy truck in a previous study [6].)

Typical driving situations that need to be considered in the design of headway control systems include:

- closing-in on a slower moving vehicle.

- a sudden change in speed by the preceding vehicle.

- a vehicle merging at short range into the path of the headway-controlled vehicle.

- and, operating on hills and curves.

Factors that influence the performance capabilities of headway control systems include control authority (that is, the deceleration and acceleration capabilities available to the control system) and sensor characteristics such as maximum reliable range and effective or swept beam width for detecting preceding vehicles. Matters related to sensor capabilities are not treated here. The paper uses the closing-in and sudden speed change situations as a basis for comparing the performance attained using different types of headway control algorithms. 
Each controller was analyzed under similar conditions and constraints. Specifically, the same vehicle and engine model was used for all the simulations. The vehicle dynamics model includes differential equations for (1) longitudinal acceleration and velocity with forces due to tire slip (traction), uphill or downhill grades, rolling resistance, aerodynamic drag, and retarder capability; (2) dynamic engine torque as a function of engine speed and accelerator setting; and (3) engine speed as influenced by inertias, efficiencies, and torque losses in the engine and drivetrain [7].

Since heavy trucks have very little natural retardation due to rolling resistance, aerodynamic drag, and engine losses, they need a supplemental source of retardation to perform speed and/or headway control satisfactorily. In this paper it is assumed that a retarder with a power capability of $250 \mathrm{hp}(192,500 \mathrm{ft} \mathrm{lb} / \mathrm{sec})$ is available for use in slowing down when the accelerator $(\delta a)$ is at its zero position $(\delta a=0)$. The foundation brakes were not used in this analysis. The control authority of the system was limited by the power of the engine at full accelerator position $(\delta \mathrm{a}=1)$ and by the level of retardation available from natural retardation sources plus the retarder. For a fully laden heavy truck, this means a maximum deceleration capability of approximately $0.05 \mathrm{~g}$ at speeds from $40 \mathrm{mph}(58.7 \mathrm{ft} / \mathrm{sec}, 66 \mathrm{~km} / \mathrm{hr})$ to $60 \mathrm{mph}$ $(88 \mathrm{ft} / \mathrm{sec}, 100 \mathrm{~km} / \mathrm{hr})$ when $\delta \mathrm{a}=0$.

\section{CONTROL ALGORITHMS AS EMPLOYED IN THE STUDY.}

\subsection{Fuzzy Logic}

Fuzzy logic has been widely used for the design of feedback control systems since the late 1980's. The major advantage of fuzzy logic is its ability to combine numerical and linguistic information. In other words, human knowledge can be easily incorporated into the control design process. For this paper, a fuzzy control algorithm has been designed by a graduate student who does not have extensive automatic control background. A standard fuzzy control system structure is used, which includes a fuzzifier, an inference engine, and a defuzzifier. The fuzzy rules in the inference engine are obtained from the designer's intuition as a human driver (not as an engineer). The input signals into the fuzzifier are headway (in seconds) and range rate (in fps), and the output from the defuzzifier is the throttle value (between 0 and 1).

The membership functions of both headway and range rate variables are divided into five sets: NL (negative large), NS (negative small), ZE (zero), PS (positive small) and PL (positive large). The range variable is centered around the desired headway time $(2.0 \mathrm{sec})$ and the range rate around 0 . The selection of the values for the membership functions are obtained manually.

A fuzzy associative matrix (FAM) was also created based on the number of membership sets for each variable. A representative FAM, corresponding to five range rate sets and five range sets, is shown in Table 1. The elements of the matrix, which are used as weighting factors for the output of fuzzy rules, are set by determining what magnitude of throttle control action is needed when headway and range rate lie fully within the sets associated with each cell in the FAM. For 
example, when both variables are at the desired values (headway set $=\mathrm{ZE}$ (located around $2 \mathrm{sec}$ ) and range rate $=\mathrm{ZE}$ (located around 0 ), (that is, FAM cell $=(2,2)$ ), the current throttle value should be maintained. Thus a value of 1 is entered in the cell. When the range rate is in the NL set and the headway is in the NL set, the throttle must be significantly reduced so a zero is entered in the $(0,0)$ cell. The FAM produced is shown in Table 1.

Table 1. FAM of the fuzzy rules

\begin{tabular}{|c|c|ccccc|}
\cline { 3 - 7 } \multicolumn{2}{c|}{} & \multicolumn{5}{|c|}{ Headway } \\
\cline { 3 - 7 } \multicolumn{2}{c|}{} & NL & NS & ZE & PS & PL \\
\hline \multirow{3}{*}{ Range } & NS & 0 & 0 & 0.2 & 0.5 & 1 \\
Rate & 0 & 0.2 & 0.5 & 1 & 1.4 \\
& ZE & 0.2 & 0.5 & 1 & 1.4 & 1.7 \\
& PS & 0.5 & 1 & 1.4 & 1.7 & 1.9 \\
& PL & 1 & 1.4 & 1.7 & 1.9 & 2.1 \\
\hline
\end{tabular}

Having calculated truth values reflecting membership in the sets of both variables, fuzzy rules of the following form are used to compute an appropriate throttle value: If range/velocity belongs to headway set $\mathrm{A}$, and range rate belongs to range rate set $\mathrm{B}$ then rule output value equals the smaller of the two truth values for sets $\mathrm{A}$ and $\mathrm{B}$ multiplied by the corresponding FAM element (B, A).

A desired throttle value is obtained through an estimation algorithm which estimates the throttle value that would be necessary to achieve the current speed of the lead vehicle. The total throttle output value (throttle setting) is the product of the desired throttle value and the summation of the rule output values for the fuzzy rules which are activated at any one time (i.e. for which neither of the conditions has a truth value of 0 ). The range of the throttle setting is limited to the interval between 0.0 (fully closed) and 1.0 (fully open). The allowable amount of change in the throttle setting per time step is limited by the dynamics of the actuator. Finally, a small integration action is added to ensure zero steady-state error in headway.

\section{2 $\mathrm{H}_{\infty}$ Optimal Control}

Another control design methodology which has attracted a lot of attention recently is the $H_{\infty}$ feedback control theory. Robustness characteristics can be guaranteed provided that the control problem is formulated properly. One of the major limitations of the $H_{\infty}$ control theory is that it can only be applied to linear time invariant systems. The truck dynamics are highly nonlinear, and therefore they must be linearized before the $H_{\infty}$ control theory can be applied. The $H_{\infty}$ control algorithm has been designed based on the plant block diagram shown in Figure 1. In this figure, $\mathrm{x}(\mathrm{t})$ is the state vector, $\mathrm{w}(\mathrm{t})$ is the external disturbance signal (e.g. lead vehicle speed and road gradient angle), and $\mathrm{z}(\mathrm{t})$ is the penalized error vector (e.g., accelerator control setting and range error).

The $H_{\infty}$ control closes the loop in between the measured output signal y(t) (range) and calculates the control signal $\mathrm{u}(\mathrm{t})$ (engine control setting) in an optimal $\left(H_{\infty}\right)$ sense. The controller is first designed in the continuous-time region, and then discretized using a zero-order-hold approximation. 


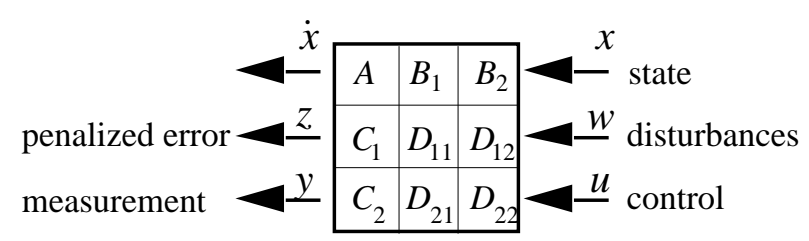

Figure 1. State space form for the $\mathrm{H}_{\infty}$ control design

The plant linearization was conducted through extensive open loop step response simulations. It was found that although the plant dynamics are nonlinear in nature, the dynamics are almost linear when we limit our scope to a short time scale (e.g. within 1-2 seconds) and when the vehicle speed does not vary too much. Under these circumstances, the relationship between the step change in engine control $\mathrm{u}(\mathrm{t})$ and the vehicle acceleration is almost linear. This linear relationship was used in our $H_{\infty}$ control design. Because of the fact that the linearized plant may be different from the true vehicle response, a small integration action is added to ensure that the range converges to the desired value at steady-state tracking. A simple anti-windup mechanism is used to ensure that the integration action does not grow unchecked when the throttle saturates.

From simulations, it was found that the truck dynamics can be approximated by the following first-order relationship:

$$
\frac{a}{u} \approx \frac{K}{1+\tau s}
$$

where $\mathrm{a}$ is acceleration, and $\mathrm{u}$ is the throttle input. $\mathrm{K}=1.07$ and $\tau=0.13$ were obtained based on a $80,000 \mathrm{lb}$ truck and $400 \mathrm{hp}$ engine within the range of possible speeds in the highest gear. In order to obtain good tracking performance, it is necessary to design the controller considering the possible disturbances from lead vehicle and road gradient. In state-space form, the truck dynamics are:

$$
\frac{d}{d t}\left[\begin{array}{l}
l \\
v \\
a
\end{array}\right]=\left[\begin{array}{ccc}
0 & 1 & 0 \\
0 & 0 & 1 \\
0 & 0 & -1 / \tau
\end{array}\right]\left[\begin{array}{l}
l \\
v \\
a
\end{array}\right]+\left[\begin{array}{cc}
0 & 0 \\
0 & 0 \\
-g & 0
\end{array}\right]\left[\begin{array}{c}
\gamma \\
l \text { lead }
\end{array}\right]+\left[\begin{array}{c}
0 \\
0 \\
K / t
\end{array}\right] u
$$

where 1 is the traveled distance, $\mathrm{v}$ is the vehicle speed, $\gamma$ is road gradient, $\mathrm{g}$ is gravity constant, and $l_{\text {lead }}$ is the distance traveled by the lead vehicle. To design an output feedback scheme, which minimizes the range error under limited control effort, we have selected

$$
\begin{aligned}
& C_{1}=\alpha\left[\begin{array}{ccc}
-1 & 0 & 0 \\
0 & 0 & 0
\end{array}\right] \quad D_{11}=\alpha\left[\begin{array}{ll}
0 & 1 \\
0 & 0
\end{array}\right] \quad D_{12}=\alpha\left[\begin{array}{l}
0 \\
\sigma
\end{array}\right] \\
& C_{2}=\left[\begin{array}{lll}
-1 & 0 & 0
\end{array}\right] D_{21}=\left[\begin{array}{ll}
0 & 1
\end{array}\right] \quad D_{22}=0
\end{aligned}
$$

The parameters $\alpha$ and $\sigma$ can be tuned for performance trade-off. The values used in the simulations are 0.005 and 10 , respectively.

\subsection{H\&S (Headway and Speed Controllers) Control by Objectives}

$\mathrm{H} \& \mathrm{~S}$ control employs a strategy that consists of a primary control objective and a subordinate control loop. The primary objective, which pertains to headway range, 
is to bring the vehicle to a desired range behind a preceding vehicle. The subordinate loop is a speed (velocity) control loop, whose purpose is to achieve the speed needed to satisfy the headway objective.

The control strategy of the primary objective is based on choosing a headway dynamics surface. The dynamics surface, used in this paper, is described by a linear relationship between range, its derivative (range-rate), and the desired range (which is a function of the speed of the preceding vehicle). Range and range-rate information are used along with the equation for the dynamics surface to generate a speed command to the velocity control loop. The velocity control loop uses the speed command to control the engine power output, and thereby to adjust vehicle speed as needed to force the system towards the headway dynamics surface.

In the H\&S control, the dynamics of closing to the desired range are determined primarily by a preview time constant $(\mathrm{T})$ that is part of the headway dynamics surface. The subordinate velocity control loop, within the bounds of constraints on maximum available acceleration and deceleration, responds rapidly enough so that speed adjustments are made quickly compared to headway adjustments. The goal is to set the preview time constant of the vehicle dynamics surface so that there is sufficient time for the maximum available deceleration or acceleration to control headway range within acceptable bounds [3].

The equations and constants used here in the H\&S control are summarized as follows:

- For stating the control objective with regard to the desired vehicle motion:

$$
\mathrm{T} \mathrm{dR} / \mathrm{dt}+\mathrm{R}-\mathrm{R}_{\mathrm{h}}=0
$$

where $R_{h}=T_{h} \cdot V_{p}$ and $T=10$ sec and $T_{h}=2$ sec.

- For evaluating the error $\mathrm{e}_{\mathrm{V}}$ with respect to satisfying the control objective:

$$
\mathrm{e}_{\mathrm{V}}=\mathrm{dR} / \mathrm{dt}+\left(\mathrm{R}-\mathrm{R}_{\mathrm{h}}\right) / \mathrm{T}=\mathrm{Vc}-\mathrm{V}
$$

where $\mathrm{Vc}$ is the command to the velocity control loop.

- The subordinate velocity control loop is based on a modified sliding mode [8] using:

$$
\mathrm{S}=\mathrm{Tv} \mathrm{de}_{\mathrm{v}} / \mathrm{dt}+\mathrm{e}_{\mathrm{v}}=0
$$

where the derivative time constant $\mathrm{Tv}=0.8 \mathrm{sec}$.

- The vehicle/engine combination (the plant) is approximated in the controller as follows:

$$
\mathrm{m}^{\prime} \mathrm{dV} / \mathrm{dt}=\delta \mathrm{a} F \mathrm{Fa}^{\prime}-\mathrm{Fr}^{\prime} \text { - Faero' - G'W' }
$$

where the primes on terms indicate estimates used in the controller, and $\delta a$ is the accelerator ("throttle") position $(0 \leq \delta \mathrm{a} \leq 1), \mathrm{Fa}$ is the maximum drive force available from the engine-transmission-tire combination, $\mathrm{Fr}$ is the rolling resistance, Fareo is the aerodynamic drag, and $\mathrm{G}$ is positive if the grade is uphill, $\mathrm{m}$ is the mass and $\mathrm{W}=\mathrm{m} \mathrm{g}$ where $\mathrm{g}$ is the gravitational constant.

- In the controller, $\mathrm{Fa}$ is represented by:

$$
\mathrm{Fa}^{\prime}=\mathrm{Pe}^{\prime} / \mathrm{V}
$$

where Pe' represents the power of the engine at full accelerator position $(\delta a=1)$.

- The form of the modified sliding mode control, including limiting $\delta$ a so that $0 \leq \delta a \leq 1$, is as follows: 


$$
\delta a=\text { Sat } 0,1\left[\delta^{\wedge}+\delta^{*}\right]
$$

where the saturation function, Sat $0,1[\cdot]$, has saturation limits at 0 and 1 .

- Estimating that $\mathrm{dVc} / \mathrm{dt} \approx 0$ and using equations 2, 3, 4, and 5 yields a feedback linearization type of control term of the form:

$$
\delta^{\wedge}=\left(\mathrm{V} / \mathrm{Pe}^{\prime}\right)\left[\left(\mathrm{W}^{\prime} / \mathrm{g} \text { Tv }\right) \mathrm{e}_{\mathrm{V}}+\mathrm{Fn}^{\prime}\right]
$$

where Fn' = Fr' + Fareo' + G'W'.

- Equation 7 is evaluated using:

$\mathrm{Fr}^{\prime}=0.01 \mathrm{~W}^{\prime} \mathrm{lbs}$

Fareo' $=800(\mathrm{~V} / 88)^{2} \mathrm{lbs}(800 \mathrm{lbs}$ at $88 \mathrm{ft} / \mathrm{sec}, 60 \mathrm{mph})$

$\mathrm{G}^{\prime}=0$ (level road)

$\mathrm{W}^{\prime}=80,000 \mathrm{lbs}$ (Examination of 7 indicates that the higher $\mathrm{W}^{\prime}$, the smaller $\mathrm{e}_{\mathrm{V}}$ needs to be to achieve steady following at constant speed.)

- To compensate for estimation errors and to add to the robustness of the control, a saturation function, providing a boundary layer, is used rather than the classical signum function; viz.,

$$
\delta^{*}=\mathrm{Kp} \mathrm{Sat}_{-1,1}\left[\mathrm{e}_{\mathrm{V}} / \mathrm{p}\right]
$$

where $\mathrm{p}=0.2 \mathrm{ft} / \mathrm{sec}$ and $\mathrm{Kp}=0.2$ (meaning up to $\pm 20 \%$ change in throttle setting due to this term). In its linear range, $\delta^{*}=1.0 \mathrm{e}_{\mathrm{V}}$.

The control equations and associated constants for the $\mathrm{H} \& \mathrm{~S}$ control were held fixed during the simulation runs thereby allowing an assessment of the level of robustness of the H\&S control. Clearly more quantities could be measured or an adaptive scheme could be used to reduce the errors. Also a small amount of integral control could be added to reduce steady state errors if very accurate control of range were needed for some special reason.

\section{EXAMPLE SIMULATION RESULTS}

Qualitative and quantitative comparisons of the performance of the three types of controllers may be extracted from the following simulation results that involve speed changes from $50 \mathrm{mph}(73.3 \mathrm{ft} / \mathrm{sec}, 22 \mathrm{~m} / \mathrm{sec})$ to $40 \mathrm{mph}(58.7 \mathrm{ft} / \mathrm{sec}, 17.6 \mathrm{~m} / \mathrm{sec})$. The closing-in runs start from a range of $250 \mathrm{ft}$ with a preceding vehicle travelling $10 \mathrm{mph}$ slower than the headway-controlled vehicle. The tracking runs start with both vehicles travelling at the same speed. Then the preceding vehicle slows from 50 $\mathrm{mph}$ to $40 \mathrm{mph}$ with a deceleration of $0.1 \mathrm{~g}$.

To examine the basic performance characteristics of the controllers in their nominal configurations, two baseline cases were studied. In the baseline cases, the vehicle was equipped with a $350 \mathrm{hp}$ engine and it weighed 60,000 lbs. The closingin and tracking maneuvers were performed on level ground in the baseline cases.

Figure 2 shows the range time histories and the range versus range rate phase plane trajectories for each of the three controllers during closing-in under the baseline conditions. Examination of the time histories shows that the systems take from 30 to 40 seconds to approach steady state with the $H \& S$ controller taking longer than the $\mathrm{H}_{\infty}$ controller. The vehicle under the $\mathrm{H} \& \mathrm{~S}$ control does not go to smaller than its steady state range. The vehicle under $\mathrm{H}_{\infty}$ control has a very small amount of under 
range behavior, while the vehicle with fuzzy control undershoots its final range noticeably.
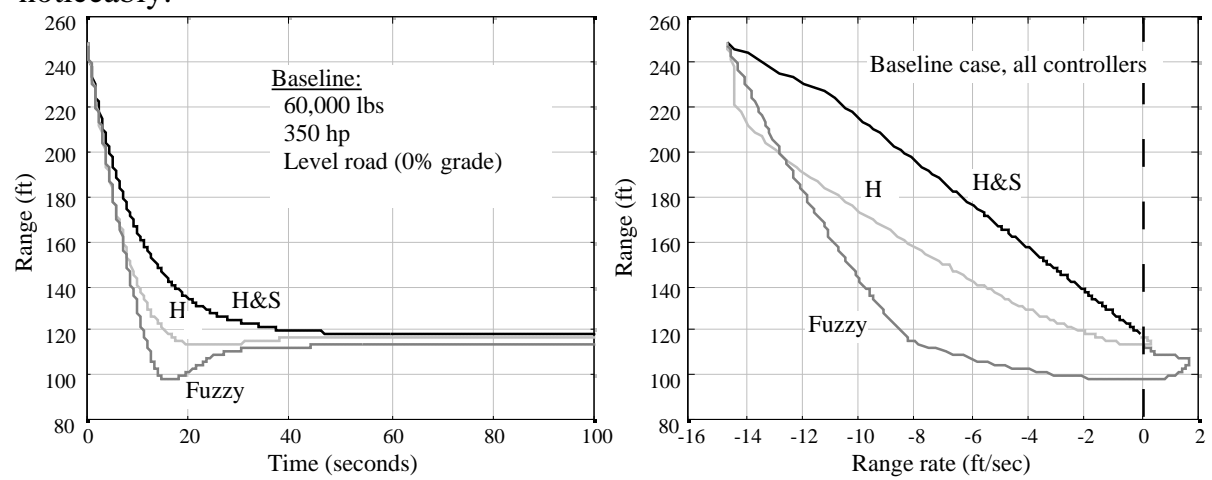

Figure 2. Baseline closing-in, range time histories and phase plane trajectories

The range versus range rate diagram in Figure 2 shows differences in how the control strategies operate. For the H\&S controller, the straight-line trajectory to the final range for following at $\mathrm{T}_{\mathrm{h}}$ is simply the control objective function, $\left(\mathrm{T} d R / d t+R=R_{h}\right)$. Since the control action starts below the objective function, the vehicle simply coasts down in speed until the trajectory reaches the objective function and then the subordinate sliding control keeps the trajectory on the objective function with unobservable error in the figure. Since the objective function represents a first order linear differential equation with a time constant of 10 seconds, the time history of $\mathrm{R}$ is approximately an exponential function once the vehicle's trajectory in the phase plane fits the objective function.

In comparison, the $\mathrm{H}_{\infty}$ control quickly goes through a few control iterations before it establishes a trajectory that takes the vehicle to the desired range with very small amounts of undershoot in $\mathrm{R}$ and overshoot in $\mathrm{dR} / \mathrm{dt}$. Once it gets organized the $\mathrm{H}_{\infty}$ control provides a smooth transition to the desired headway situation. There is only a very small amount of acceleration needed at the end to bring the speed of the headway-controlled vehicle up to the speed of the preceding vehicle.

In contrast to the other controllers, use of the Fuzzy controller produces a fairly large undershoot in range which entails a fair amount of acceleration to bring the range rate to zero at the end of the closing-in maneuver. (See Figure 2.) 


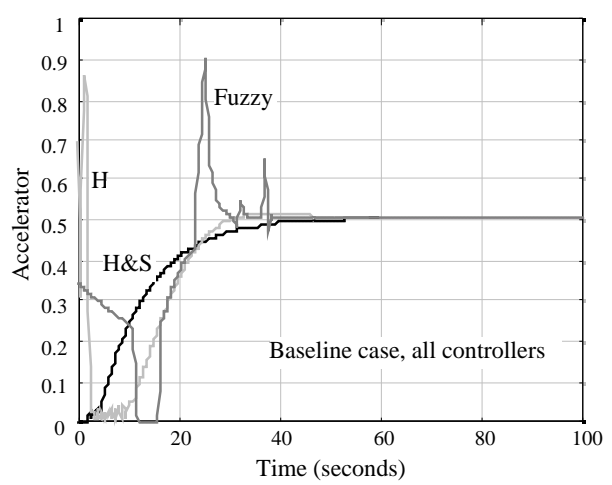

Figure 3. Baseline closing in, accelerator time histories

The specific differences between the control actions of the controllers can be seen by examining Figure 3. Clearly the fuzzy system has large amplitude excursions of the control near the end of the maneuver indicating that perhaps more tuning would be in order to perfect the fuzzy system. Except for some rather large excursions of the throttle at the beginning of the maneuver, the $\mathrm{H}_{\infty}$ system comes from near zero control setting to $\delta a \approx 0.5$ in a smooth manner. The control action of the H\&S system is exponentially smooth since the control authority of the system and the quickness of the sliding control are more than adequate for most of the time during this closing-in maneuver.

When tracking a preceding vehicle that makes a $10 \mathrm{mph}$ change in speed, the range time histories are characterized by a sudden drop in range followed by corrective actions that differ, depending upon the controller involved. This is a much more severe maneuver than the closing-in maneuver. The initial range is $147 \mathrm{ft}$ as compared to $250 \mathrm{ft}$ for the closing-in case, therefore the responsiveness of the controller is crucial. The time histories presented in Figure 4 show that the Fuzzy controller allows a significantly larger range undershoot than the other two controllers. The phase plane trajectories in Figure 4 show that the H\&S system reaches its objective function (straight-line appearance in Figure 4) before $\mathrm{dR} / \mathrm{dt}$ reaches zero. However, the $\mathrm{H}_{\infty}$ and the Fuzzy systems allow dR/dt to overshoot the $\mathrm{dR} / \mathrm{dt}=0$ line by 1 and $2 \mathrm{ft} / \mathrm{sec}$, respectively. 

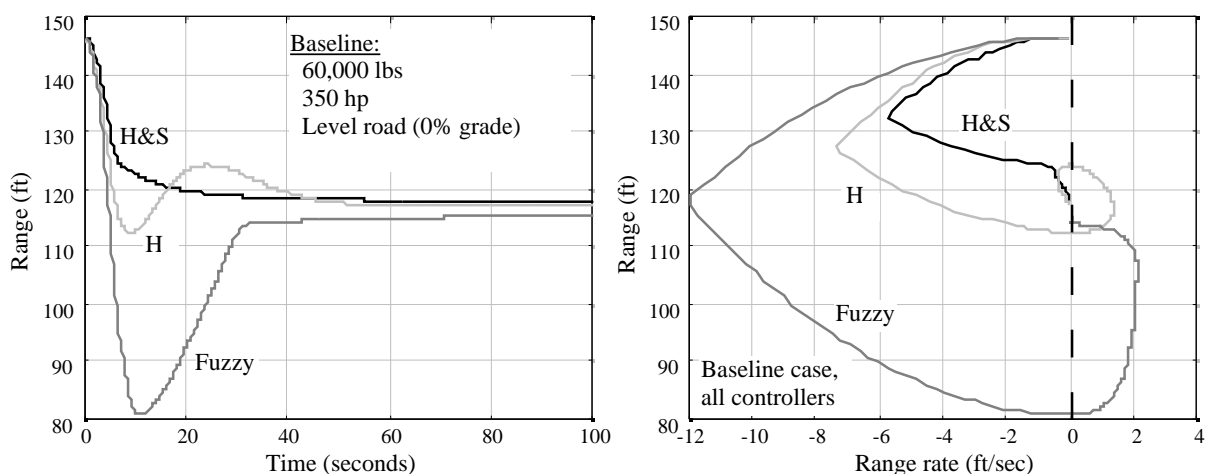

Figure 4. Baseline tracking a 10mph speed change, range time histories and phase plane trajectories

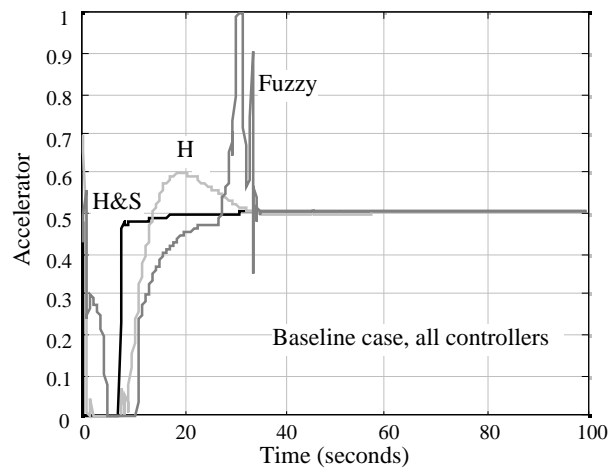

Figure 5. Tracking a 10mph speed change, accelerator time histories

These results can be explained by examining the control actions of the controllers. Examination of Figure 5 shows that the H\&S control action is characterized by a sudden drop of the accelerator control, until about 7 seconds, followed by a rapid rise to nearly the final required value for the control setting. This means that the system reaches its objective function in about 7 seconds and then follows the objective line (the straight line section in the phase plane diagram of Figure 4) with only gradual changes in the throttle setting. Although with greater variations, the $\mathrm{H}_{\infty}$ system does a similar control action with some overshoot of the final control setting. As in the closing-in case, the Fuzzy system has a few large control excursions as the system approaches its final steady value.

The results shown in Figures 2 through 5 show that the $\mathrm{H} \& \mathrm{~S}$ and $\mathrm{H}_{\infty}$ controllers work well. However, the Fuzzy controller does not appear to achieve what might be accomplished after a period of further tuning. Nevertheless, results for the fuzzy system are included in the cases that follow.

In order to challenge the robustness of the controllers, the closing-in and tracking maneuvers were studied in the following situations which include perturbations from the baseline condition as well as the baseline condition itself:

(1) vehicle weighs $34,000 \mathrm{lb}$ (empty) as compared to 60,000 lb (baseline) 
(2) vehicle is operating on a $2 \%$ downgrade (down slope $=-0.02$ radians)

(3) vehicle has a $250 \mathrm{hp}$ engine as compared to $350 \mathrm{hp}$ for the baseline case.

(4) baseline case 60,000 lb, $350 \mathrm{hp}$, level ground (see Figures 2 through 5)

(5) vehicle has a $450 \mathrm{hp}$ engine.

(6) vehicle is operating on a $+2 \%$ upgrade.

(7) vehicle weighs $80,000 \mathrm{lb}$ corresponding to the fully laden legal weight.

The results of the robustness study are tabulated in terms of three measures of performance. These are (1) the minimum range during the maneuver indicating the undershoot in range (see Table 2), (2) the maximum value of $\mathrm{dR} / \mathrm{dt}$ during the maneuver indicating the overshoot in range rate (see Table 3), and (3) the length of time for $|\mathrm{dR} / \mathrm{dt}|$ to reach and stay less than $1 \mathrm{ft} / \mathrm{sec}$ indicating the settling time needed to establish following (see Table 4).

Table 2. Minimum range ( $\mathrm{ft}$ )

\begin{tabular}{|l|l|l|l|l|l|l|}
$34 \mathrm{~K}$ & $(-.02)$ & $250 \mathrm{hp}$ & Baseline & $450 \mathrm{hp}$ & $(+.02)$ & $80 \mathrm{~K}$ \\
\hline
\end{tabular}

\begin{tabular}{|l|l|l|l|l|l|l|l|l|l|}
\hline Close & Track & & & & & \\
\hline
\end{tabular}

\begin{tabular}{|l|l|l|l|l|l|l|l|l|l|l|l|l|l|l|}
\hline H\&S & 118 & 118 & 116 & 99 & 119 & 119 & 118 & 118 & 118 & 118 & 121 & 121 & 119 & 109 \\
\hline Hinf & 113 & 117 & 110 & 92 & 115 & 113 & 113 & 112 & 113 & 114 & 117 & 117 & 113 & 100 \\
\hline Fuzz & 117 & 101 & 39 & 36 & 98 & 81 & 98 & 81 & 101 & 83 & 110 & 102 & 84 & 66 \\
\hline
\end{tabular}

Table 3. Maximum $|\mathrm{dR} / \mathrm{dt}|(\mathrm{ft} / \mathrm{sec})$

\begin{tabular}{|l|l|l|l|l|l|l|}
$34 \mathrm{~K}$ & $(-.02)$ & $250 \mathrm{hp}$ & Baseline & $450 \mathrm{hp}$ & $(+.02)$ & $80 \mathrm{~K}$ \\
\hline
\end{tabular}

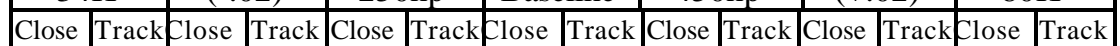

\begin{tabular}{|l|l|l|l|l|l|l|l|l|l|l|l|l|l|l|}
\hline $\mathrm{H} \& \mathrm{~S}$ & 0 & 0 & 0 & 1.60 & 0 & 0 & 0 & 0 & 0 & 0 & 0 & 0 & 0 & 0.90 \\
\hline
\end{tabular}

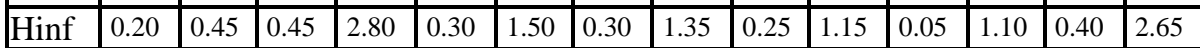

\begin{tabular}{|l|l|l|l|l|l|l|l|l|l|l|l|l|l|l|}
\hline Fuzz & 0.05 & 1.75 & 3.55 & 2.00 & 1.65 & 1.95 & 1.70 & 2.10 & 1.60 & 2.20 & 0.60 & 3.90 & 1.95 & 2.10 \\
\hline
\end{tabular}

Table 4. Time till $|\mathrm{dR} / \mathrm{dt}|<1 \mathrm{ft} / \mathrm{sec}$ continuously thereafter (sec.)

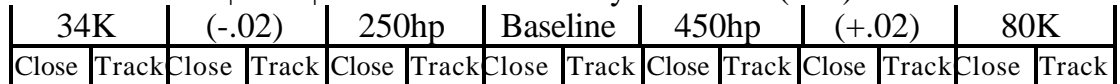

\begin{tabular}{|l|l|l|l|l|l|l|l|l|l|l|l|l|l|l|}
\hline $\mathrm{H} \& \mathrm{~S}$ & 25.5 & 12.5 & 25.0 & 17.0 & 25.0 & 7.0 & 25.0 & 7.0 & 25.0 & 7.0 & 24.5 & 10.0 & 25.0 & 9.0 \\
\hline
\end{tabular}

\begin{tabular}{|l|l|l|l|l|l|l|l|l|l|l|l|l|l|l|}
\hline Hinf & 19.0 & 7.5 & 19.5 & 24.5 & 18.0 & 18.0 & 18.5 & 17.0 & 18.5 & 15.5 & 19.0 & 14.5 & 18.5 & 23.0 \\
\hline
\end{tabular}

\begin{tabular}{|l|l|l|l|l|l|l|l|l|l|l|l|l|l|l|}
\hline Fuzz & 17.0 & 14.5 & 50.0 & 88.5 & 25.5 & 31.0 & 25.0 & 30.5 & 23.0 & 28.5 & 42.5 & 81.5 & 37.0 & 41.0 \\
\hline
\end{tabular}

For the particular closing-in and tracking maneuvers used here, the steady range during following at the end of the maneuvers should be approximately $117 \mathrm{ft}$ for $\mathrm{T}_{\mathrm{h}}=2 \mathrm{sec}$ and a speed of $40 \mathrm{mph}(58.7 \mathrm{ft} / \mathrm{sec}, 24 \mathrm{~km} / \mathrm{hr})$. The entries in Table 2 for minimum range show that the fuzzy system has values of minimum range that are considerably less than 117 for all the situations except for closing-in with the vehicle weighing $34,000 \mathrm{lb}$ or with a two percent upgrade. In particular, the situation corresponding to tracking a $10 \mathrm{mph}$ speed change on a two percent downgrade is a difficult challenge for the fuzzy system as indicated by a minimum range of $36 \mathrm{ft}$.

Although the $\mathrm{H} \& \mathrm{~S}$ and the $\mathrm{H}_{\infty}$ controllers do much better than the Fuzzy controller, they also have their greatest undershoots for the downhill tracking situation on a two percent downgrade. The H\&S controller tends to have the least undershoot since it only undershoots when the control authority in deceleration is not sufficient to prevent it. The results for the $\mathrm{H} \& \mathrm{~S}$ and the $\mathrm{H}_{\infty}$ controllers are 
compatible with the observations made in connection with the discussions of the baseline cases and Figures 2 through 5.

Examination of the maximum value of $\mathrm{dR} / \mathrm{dt}$ (see Table 3 ) in the perturbation situations shows no overshoot in $\mathrm{dR} / \mathrm{dt}$ for the H\&S system except in the tracking maneuvers on a two percent downgrade or when the vehicle weighs $80,000 \mathrm{lb}$. The other controllers do not allow a large amount of overshoot in $\mathrm{dR} / \mathrm{dt}$, meaning that the headway controlled vehicles only need to accelerate a small amount in order to catch up to the speed of the preceding vehicle after they have gone through minimum range.

The most significant entries in Table 4 pertain to situations in which the Fuzzy controller has had difficulties in finding the appropriate throttle setting. As might be expected the most difficult situation for all of the controllers is the two percent down grade. Given that the control authority for deceleration is only about $0.05 \mathrm{~g}$ and the two percent downgrade means a loss of $0.02 \mathrm{~g}$ of deceleration, the control systems are operating with only $0.03 \mathrm{~g}$ deceleration on this downgrade. This represents a large perturbation in the nature of the system compared to the system used for the baseline condition.

An overall examination of Tables 2, 3, and 4 indicates that the controllers operate fairly well under all of the perturbations except the downhill case. Given the limited acceleration/deceleration control authority, it is clear that grades impose the most challenging disturbances. However, perturbation to the vehicle weight did not cause significant problems. This indicates that a control system that was designed for maximum load, can be expected to function satisfactorily under lighter loads as well.

Figures 6 and 7 provide further information on the performances of the controllers when their control authority is changed due to grade. The details of the performances of the systems are shown in the range time histories and the phase plane trajectories presented in these figures. It took over 88 seconds for the fuzzy controller to find a solution for steady motion on the two percent downgrade. See Figure 6. (This is a long hill.) None of the controllers can prevent undershoot. As seen in the phase plane diagram, the H\&S controller follows "up" the objective function to reach the desired range. This means that the headway-controlled vehicle needs to travel faster than the preceding vehicle. 

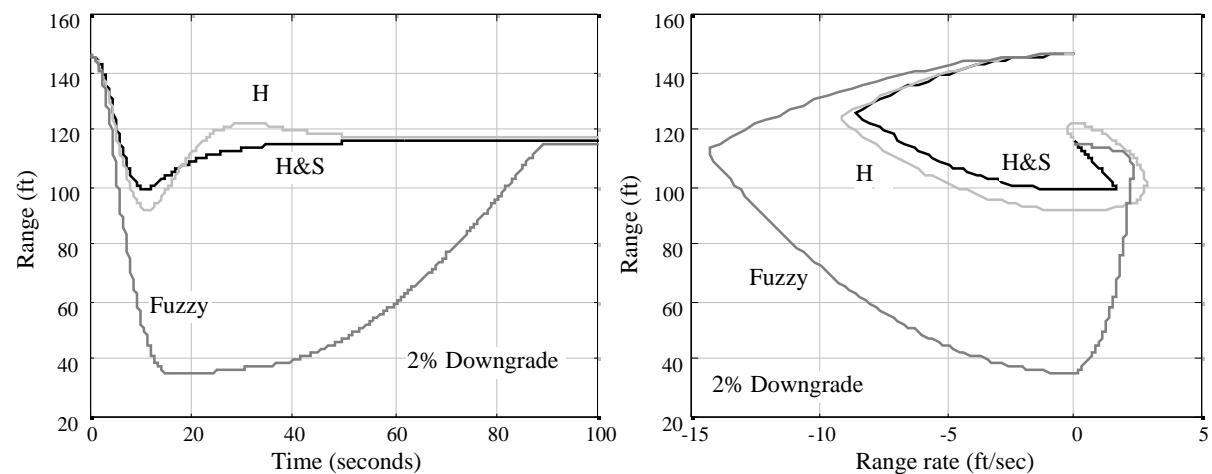

Figure 6. $2 \%$ downgrade tracking a $10 \mathrm{mph}$ speed change, range time histories and phase plane trajectories
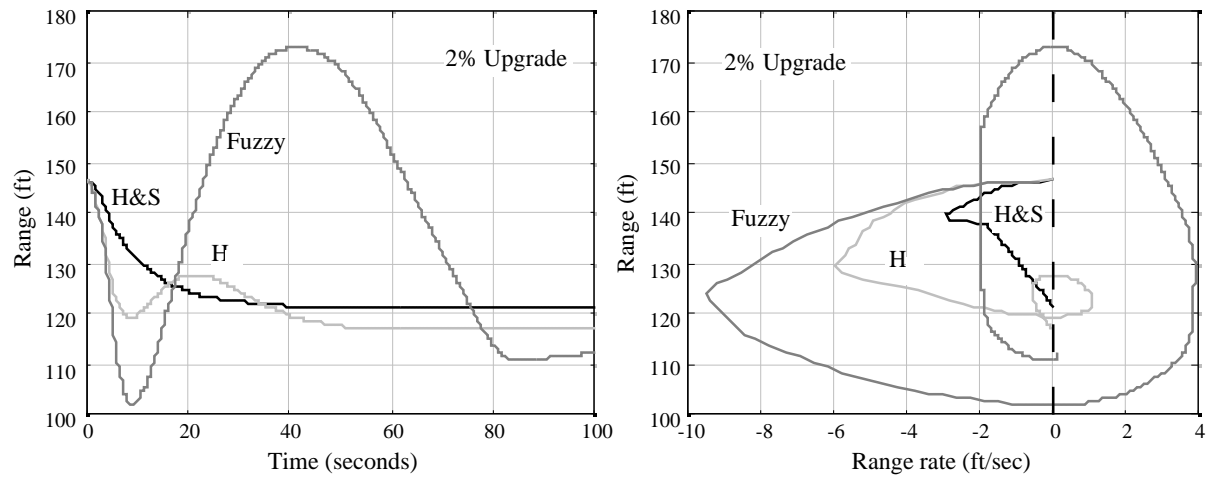

Figure 7. $2 \%$ upgrade tracking a $10 \mathrm{mph}$ speed change, range time histories and phase plane trajectories

The uphill case (Figure 7), although not a challenge to any of the systems, illustrates some interesting points. The system with the fuzzy controller goes through large excursions in range. The phase plane trajectory for the $\mathrm{H}_{\infty}$ system shows an interesting loop as it "misses" the desired range on its first pass through $\mathrm{dR} / \mathrm{dt}=0$. This probably is not a major factor but some drivers might not like the feeling of speeding up just when it seems that they are where they want to be. The $\mathrm{H} \& \mathrm{~S}$ controller has a higher steady state range because it needs to have a small steady state error to achieve the throttle setting needed to maintain the proper speed. Some integral control could be added if this were a concern. Clearly, there are details that could be considered in perfecting these systems.

\section{CONCLUDING STATEMENTS AND COMPARISONS}

Figure 8 shows the functional elements of each of the three types of controllers placed in the context of the overall headway control system. The output of each controller is the accelerator setting $\delta$ and each controller receives inputs from the sensors. 


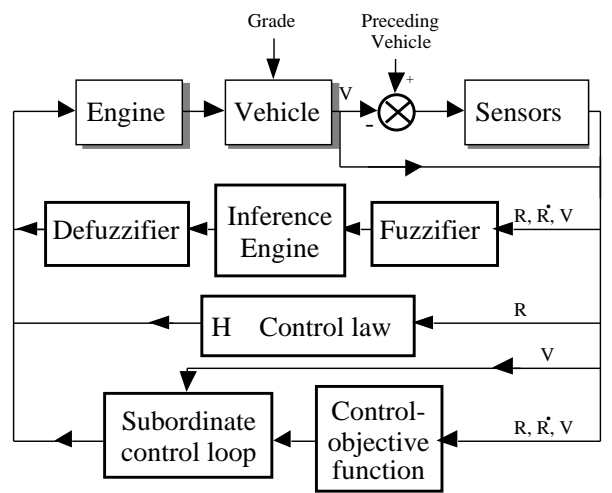

Figure 8. Summary of three headway controllers

However, the requirements for implementing the controllers are very different and, as has been shown quantitatively by Figures and Tables, the performance of the overall system differs considerably depending upon the controller used.

The fuzzy controller involves an on-line evaluation of the fuzzy sets, and the implementation of the fuzzy inference rules and the defuzzifier. This is a relatively complicated implementation compared to the other controllers. In addition, the control designer may be uncertain as to what procedure to use to improve the performance of the overall system.

The resulting controller may not be robust. The control actions may amount to rough rather than smooth control inputs. In our case, the fuzzy controller used knowledge of the vehicle's accelerator/speed characteristics. However in general, fuzzy control requires little knowledge of the vehicle.

For the $\mathrm{H}_{\infty}$ optimal control there are complicated design calculations to be done off-line, but there are control design programs for doing these calculations in a routine manner, e.g. [9]. The resulting control law is very easy to implement. For example, in this case the control algorithm (expressed in Z-transform notation) is as follows:

$\frac{\delta}{\mathrm{R}-\mathrm{R}_{\mathrm{h}}}=\frac{2.2134 \cdot 10^{-2} \cdot \mathrm{z}^{-1}-4.2586 \cdot 10^{-2} \cdot \mathrm{z}^{-2}+2.0455 \cdot 10^{-2} \cdot \mathrm{z}^{-3}}{1-2.8953 \cdot \mathrm{z}^{-1}+2.7881 \cdot \mathrm{z}^{-2}-0.89553 \cdot \mathrm{z}^{-3}}$

This means that the current accelerator setting is simply equal to a weighted sum of the previous three values of throttle setting plus a weighted sum of the previous three values of range error.

The $\mathrm{H}_{\infty}$ controller is robust and works well even when there are large perturbations in system characteristics. The control action is smooth. The system demonstrates some undershoot in range and some overshoot in range-rate. In order to apply the $\mathrm{H}_{\infty}$ procedure used here, one needs to develop a good linear approximation for the openloop performance of the engine-vehicle combination.

The $\mathrm{H} \& \mathrm{~S}$ control is easy to implement once the designer chooses a control objective function. The basic idea of $\mathrm{H} \& \mathrm{~S}$ control by objectives is to substitute the sensor readings into the control objective function to obtain the error $\mathrm{e}_{\mathrm{V}}$ between "what you want" and "what you have". Given a value for $\mathrm{e}_{\mathrm{v}}$, any type of control 
system may be used for forcing $\mathrm{e}_{\mathrm{V}}$ towards zero. In this case a modified slidingmode control was used to force $e_{v}$ towards zero. However, if the vehicle already has a cruise control system, it can be used as the subordinate control loop employed for obtaining a speed and range that will satisfy the control objective [4]. (The cruise control type of subordinate loop is indicated in Figure 8 by the velocity signal V which by-passes the control objective function.)

The $\mathrm{H} \& \mathrm{~S}$ control is robust. There is no undershoot in range or overshoot in range rate as long as the system is operating within the control authority limits pertaining to the engine and the available sources of retardation.

When the sliding-mode control is used, a simple non-linear model of the enginevehicle combination is needed to develop the feedback linearization part of the control setting for the accelerator. In this case, the determination of the control objective function and the subordinate control loop requires that the system designer knows (1) what the desired performance of the overall system is to be and (2) how the plant (engine-vehicle combination) works.

Finally, it can be said that the control by objective approach (which we have called "H \& S" control) can be generalized for use in many vehicle system dynamics applications. If one knows or has in mind a differential equation for the desired performance of the overall system, this equation can be used as a candidate for the control objective function.

\section{ACKNOWLEDGMENT}

This work was supported in cooperation with the Great Lakes Center for Truck and Transit Research.

\section{REFERENCES}

1. Mamdani, E.H., "Applications of Fuzzy Algorithms for Simple Dynamic Plant," Proc. IEEE, 121, no. 12 (1974), pp.1585-1588.

2. J.C. Doyle, K. Glover, P.P. Khargonekar and B.A. Francis, "State-space Solutions to Standard H_2 and H_inf Control Problems," IEEE Transactions on Automatic Control, Vol.34, No.8, pp.831-847, 1989.

3. Fancher, P. and Bareket, Z., "Evaluating Headway Control Using Range Versus Range-Rate Relationships" Vehicle System Dynamics, 23 (1994), pp. 575-596

4. Fancher, P.S., Ervin, R.D., Bareket, Z., Johnson, G.E., Trefalt, M., Tiedecke, J. and Hagleitner, W., "Intelligent Cruise Control: Performance Studies Based Upon an Operating Prototype." IVHS America Annual Meeting, IVHS America Annual Meeting. Atlanta, Georgia, 1994.

5. Chakraborty, S. and Smedley, D.G., "Adaptive Cruise Control for Heavy Duty Vehicles," Intelligent Transportation: Serving the User Through Deployment, Proceedings of the 1995 Annual Meeting of ITS AMERICA, Washington D.C., March 15-17, 1995, Vol. 1, pp.145-150.

6. Bareket, Z., Fancher, P.S., and Johnson, G., "Control of Headway Between Successive Vehicles," US ARMY TACOM DAAE07-93C-R126, Final Report, The University of Michigan Transportation Research Institute, Ann Arbor, MI, March, 1995

7. Bareket, Z., Fancher, P.S., and Johnson, G., "Investigation of the Performance of a HeadwayControl System for Commercial Vehicles," IVHS Technical Report 93-01, The University of Michigan, Jan. 1993. 
Vehicle System Dynamics, Vol.25 suppl., 1996, pp.139-151

8. Slotine, J-J.E., and Li, W.: Applied Nonlinear Control. Prentice Hall, Englewood Cliffs, New Jersey, 1991.

9. MATLAB User's Guide, The MathWorks, Inc., Cochituate Place, 24 Prime Park Way, Natick, MA, 01760, Sept. 1993. 